\title{
Marie-Claire Bichard-Thomine, Noël Du Fail Conteur
}

\section{Valerio Cordiner}

\section{OpenEdition \\ Journals}

\section{Edizione digitale}

URL: http://journals.openedition.org/studifrancesi/35603

DOI: 10.4000/studifrancesi.35603

ISSN: 2421-5856

\section{Editore}

Rosenberg \& Sellier

\section{Edizione cartacea}

Data di pubblicazione: 1 juillet 2005

Paginazione: 115-118

ISSN: 0039-2944

\section{Notizia bibliografica digitale}

Valerio Cordiner, «Marie-Claire Bichard-Thomine, Noël Du Fail Conteur», Studi Francesi [Online], 145 (XLIX | I) | 2005, online dal 30 novembre 2015, consultato il 20 avril 2021. URL: http://

journals.openedition.org/studifrancesi/35603 ; DOI: https://doi.org/10.4000/studifrancesi.35603

Questo documento è stato generato automaticamente il 20 avril 2021.

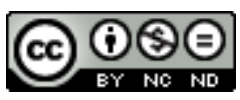

Studi Francesi è distribuita con Licenza Creative Commons Attribuzione - Non commerciale - Non opere derivate 4.0 Internazionale. 


\title{
Marie-Claire Bichard-Thomine, Noël Du Fail Conteur
}

\author{
Valerio Cordiner
}

\section{NOTIZIA}

MARIE-CLAIRE BICHARD-THOMinE, Noël Du Fail Conteur, Paris, Honoré Champion Éditeur, 2001, $627 \mathrm{pp}$.

1 I lettori e gli studiosi dell'opera di Noël Du Fail attendevano impazientemente la pubblicazione del lavoro di Marie-Claire Bichard-Thomine. La magra bibliografia dufailiana - costituita in sostanza dagli studi rigorosi, ma datati, di La Borderie e Philipot e dagli interventi lodevoli, ma settoriali, di Olivier-Martin, Milin, Sozzi, Pérouse e dei relatori del convegno rennese del 1987 - è oggi sensibilmente rinvigorita. Le vaste terre largamente inesplorate del gentiluomo della Hérissaie sono oggi nitidamente rappresentate nel tableau esaustivo di $\mathrm{M}^{\mathrm{me}}$ Bichard-Thomine. $\mathrm{E}$, anzi, la prima impressione che suscita nello specialista la lettura di questo studio voluminoso è quasi di disappunto; impressione dolorosa di essere giunti troppo tardi, quando tutto oramai è stato scritto.

Data l'ampiezza e la complessità del lavoro, senza attardarci in pur meritati encomi, cerchiamo di seguire nelle grandi linee il ragionamento che si snoda lungo le oltre 600 pagine di Noël Du Fail conteur. «Nomen omen»: nel titolo è già svelato lo sterminato settore di ricerca prescelto dalla studiosa: la presenza dei contes nell'opera narrativa del gentiluomo bretone, i legami con il retaggio medievale patrio e con la più recente lezione degli Italiani, i complessi rapporti con l'ideologia conservatrice che si esprime nei discours. Sospesa tra tradizione e innovazione, incerta tra le ragioni dell'arte e quelle della politica, l'opera di Du Fail è alla strenua ricerca di un fragile equilibrio. Questa indecisione si rivela già nei procedimenti di mise en cuvre illustrati nel I capitolo del volume di $\mathrm{M}^{\text {me }}$ Bichard-Thomine. Nei Propos rustiques, raffigurazione puntuale, ma intrisa di commossa nostalgia, della campagna bretone - luogo edenico dell'infanzia - il 
narratore Leon Ladulphi (pseudonimo trasparente di Noël Du Fail) rimane per lo più dietro le quinte, intento a spiare con compiaciuta ammirazione il concerto polifonico dei devisants contadini. La parola, affidata al collegio dei senatori rustiques, si piega docilmente agli intenti rievocativi, e a volte scrupolosamente etnologici, di questa suggestiva ricostruzione di un piccolo mondo antico oramai al tramonto. Nelle Baliverneries d'Eutrapel, pur non rinunciando all'intento mimetico della campagna bretone, Noël Du Fail si avvale con maggior frequenza del registro comico. La saggia lezione dei maestri umanisti Erasmo e Rabelais l'aveva, forse, convinto a ricorrere all'espediente della facetia per sfuggire alle critiche di esegeti malevoli. L'appassionata difesa del naturel è ora affidata non solo al consesso dei devisants, che si fa più ristretto $\mathrm{e}$ qualificato, ma anche agli interventi di un narratore più presente e loquace. Nei Contes et discours d'Eutrapel il feu seigneur de la Hérissaye, invece, declinato ogni tentativo di organizzazione razionale dell'opera, si eclissa all'orizzonte del serrato dibattito tra i conversatori. La riflessione ideologica, nella fattispecie di una disquisizione di argomento giuridico, si impossessa da subito della scena.

Il II capitolo di Noël Du Fail conteur affronta la complessa questione della definizione di genere delle raccolte dufailiane. La difficoltà dell'impresa discende da fattori concomitanti puntualmente evidenziati dalla studiosa: l'assoluta approssimazione della nozione di genere quale emerge dai trattati rinascimentali; un contesto letterario in rapida evoluzione, in cui archetipi e suggestioni si incontrano e si confondono in composti instabili e contraddittori; un frangente storico travagliato, segnato dall'instabilità politica e dalle divisioni religiose. La conseguente tendenza epocale alla bigarrure si manifesta in tutta la sua problematica ambiguità in molte raccolte di prosa breve e notamment nell'opera di Noël Du Fail, ove si incontrano le istanze conflittuali della narrazione, della descrizione, del dialogo e della riflessione. Così, dal realismo descrittivo dei Propos Rustiques si giunge, per il tramite del serrato dibattito tra i devisants, alla spiccata dimensione ideologica della raccolta della maturità. Le istanze contrapposte della moquerie e della didattica, impersonate da Eutrapel e Polygame, sono incapaci di approdare ad un momento sintetico. E, anzi, all'atmosfera unanime dei Propos Rustiques subentra una costruzione binaria articolata secondo schemi oppositivi. La scrittura dufailiana sembra rinunciare alle ambizioni mimetiche, riducendosi a mera illustrazione di una dottrina politica. Nell'impossibilità di ricondurla ad un arcitesto funzionale, non resta, dunque, che accertare ciò che l'opera di Noël Du Fail non è: sicuramente non pastorale, genere anzi ridicolizzato dal robusto realismo contadino; non facetia, di cui non si rispetta la disposizione alla brevità e all'autoreferenzialità; neanche novella, visto e considerato il conclamato rifiuto delle suggestioni della cultura cittadina e cortigiana; ma nemmeno, infine, qualcosa di simile all'innovativo esperimento montaignano, per l'incapacità a sottrarsi alla préjuditiale facétieuse, ma anche alle pastoie del dibattito politico contemporaneo. Da una raccolta all'altra l'opera di Noël Du Fail si uniforma alle tendenze evolutive della prosa breve. L'immagine del mondo agreste diventa più letteraria e ideologicamente condizionata; si attenua la dimensione orale. L'eco della formazione umanistica si riverbera più nitidamente nell'ultima raccolta, non solo attraverso la pletora di riferimenti dotti e di citazioni erudite, ma anche nel trattamento della materia narrativa e negli argomenti dibattuti nelle accese discussioni tra i devisants - che spaziano dalla giurisprudenza alla medicina, dallo studio della lingua all'interpretazione delle Scritture. Pur evidenziando puntualmente tempi e modalità di questa evoluzione interna, $\mathrm{M}^{\mathrm{me}}$ Bichard-Thomine riconosce, comunque, una vocazione costante all'opera dufailiana: ovvero l'attenzione 
rivolta al dato reale, che finisce sempre per prevalere sia sugli intenti comici, sia su quelli ideologici. La cifra della sua scrittura si rinviene, dunque, nello sforzo di dare forma e consistenza all'arte di descrivere.

Nel III capitolo l'autrice esamina la complessa struttura architettonica dell'opera. In primis, ella focalizza l'attenzione sulla presenza - tutt'altro che rituale nelle raccolte cinquecentesche - e sul ruolo, cangiante tra un'opera e l'altra, dei titoli. Se nei Propos Rustiques essi suddividono in sequenze il dialogo tra i devisants, nelle Baliverneries d'Entrapel isolano, invece, segmenti aneddotici a sé stanti; nei Contes et Discours d'Entrapel, infine, consentono di definire gli assi portanti della raccolta, indicando le tematiche trattate nei capitoli e riconducendo le narrazioni ad intenti esemplari. L'unità dei capitoli è garantita nei Propos Rustiques da un'organizzazione rigorosa della narrazione, ordinata in sequenze temporali che corrispondono a momenti di una rievocazione corale dei tempi che furono nel contesto attendibile di una veillée contadina. Nelle Baliverneries d'Entrapel si assiste ad un incipiente processo di dissolvimento della struttura narrativa; dall'uniforme organicità del consesso rusticano si liberano le voci ben distinte di tre devisants che comunicano idee, sentimenti ed istanze differenti quando non contrapposte. Questa tendenza alla bigarrure si accentua nei Contes et Discours d'Entrapel ove, in assenza di pur artificiose indicazioni spaziotemporali (e cioè di un cadre e di una cronologia), la materia narrativa si accumula in maniera caotica e discontinua, eludendo ogni tentativo di organizzazione razionale.

Come illustra il IV capitolo, il rapporto che intercorre tra titoli e capitoli si dissolve da una raccolta all'altra. Il perfetto congegno strutturante - incarnato nei Propos Rustiques dall'unità di tema e di locutore all'interno dei singoli capitoli - comincia a incepparsi nelle Baliverneries d'Entrapel sotto l'azione disgregatrice del dialogo a batôns rompus, per bloccarsi definitivamente nei Contes et Discours d'Entrapel ove l'unico, labile criterio organizzatore è costituito dallo svolgimento puramente ideale di una conversazione fittizia. Interessante è anche rilevare come, da una raccolta all'altra, mutino radicalmente lo statuto e il ruolo della narrazione. Dal grado zero dei Propos Rustiques ove, se si eccettua l'excursus di Tailleboudin, prevale uno statico descrittivismo realista - alla presenza episodica (e sempre funzionale alla mimesi) delle $B E$, al foisonnement, forse riconducibile alla lezione di Marguerite e di Des Périers, dei Contes et Discours d'Entrapel. Ma ancor di più emergono gli intenti ideologici che sussumono la narrazione, dagli aneddoti di poche righe sino alle lunghe histoires tragiques, a finalità esemplificative di una qualche teoria. Il realismo scrupoloso dei Propos Rustiques, ma anche delle Baliverneries d'Entrapel (si pensi ad esempio alla minuziosa descrizione della dimora rurale nel IV capitolo) sembra non esaurire più l'orizzonte creativo del prosatore bretone.

6 Lo studio della lingua dufailiana costituisce l'oggetto del V capitolo. L'indagine prende le mosse dalla percezione della progressiva abdicazione della parola da parte del narratore a beneficio dei suoi personaggi, sulla scorta, forse, della proliferazione in ambiente umanista del dialogo filosofico e della civile conversazione. Nei Propos Rustiques il narratore Leon Ladulphi riveste il ruolo di umile comparsa; rimasto in disparte ad ascoltare attentamente la conversazione tra i commensali della soirée rustique, egli si limita ad occasionali interventi descrittivi o più semplicemente interlocutori. Accordando generosamente la parola ai suoi devisants egli inaugura un costume cui questi ultimi ricorreranno di buon grado a favore dei loro personaggi, generando in tal modo quelle che Todorov ha definito delle piramidi narrative. Il vasto 
coro dei Propos Rustiques si riduce nelle Baliverneries d'Entrapel a soli tre elementi. Se i devisants, ad eccezione di Eutrapel, smarriscono lo spessore umano e la carnalità dei Propos Rustiques, il narratore, in veste di direttore d'orchestra, interviene assiduamente per il tramite del discorso indiretto; la polifonia vi si manifesta a livello della diegesi. Nei Contes et Discours d'Entrapel, in assenza di un narratore, dato per morto nel titolo, ci attenderemo una rinnovata fioritura del dialogo tra i devisants. E, invece, dissoltosi il décor rusticano, la dimensione schiettamente orale, che aveva contraddistinto la conversazione nelle prime raccolte, sopravvive solo nell'esuberanza verbale di Eutrapel: nei discours degli altri conversatori - e soprattutto di Poligame che, forte della riconosciuta egemonia, occupa stabilmente la scena - la propensione al monologo e i marcati intenti ideologici compromettono la naturalezza e la stessa credibilità della finzione dialogica.

Il capitolo VI analizza l'incidenza della penetrazione della cultura umanista nei dialoghi tra i personaggi dufailiani, in relazione con il concomitante processo di separazione tra la cultura popolare e quella delle élites. Così, tra una raccolta e l'altra, assistiamo ad un progressivo scivolamento dall'inflessione contadina dei commensali dei Propos Rustiques ai dotti conversari dei devisants dei Contes et Discours d'Entrapel, secondo una tendenza dominante ad armonizzare lo stile al contenuto della narrazione. Nei Propos Rustiques il realismo linguistico discende dal tono colloquiale della conversazione (che però solo episodicamente si avvale, a differenza di Rabelais, dei provincialismi). Nelle Baliverneries d'Entrapel il narratore si esprime spesso con il gergo dell'uomo di legge. Dismessa la coralità dei Propos Rustiques, emergono le individualità linguistiche dei conversatori; e, in primis, di Eutrapel che non disdegna di ricorrere, con aperte finalità parodistiche, al registro comico-scurrile, anche nella trattazione di argomenti seriosi. Nei Contes et Discours d'Entrapel, infine, zittitosi il narratore, si delineano, nell'inflessione delle loro voci discordanti, i contorni psicologici dei devisants. Nel contrasto tra la vibrante oratoria giuridica di Poligame e la verve popolana di Eutrapel traspare una spiccata propensione al mélange degli stili. Assai pronunciato è lo scarto tra contes e discours, tra la iocosa liberalitas (di osservanza erasmiana) dei primi e l'erudizione ostentata dei secondi.

8 La coloritura popolare della prosa dufailiana costituisce l'oggetto del capitolo VII. L'attenzione della studiosa si focalizza sulla presenza dei proverbi, presenza che, seppur riconducibile alla cultura popolare, è frequente nelle più fini produzioni umanistiche. L'impiego massiccio, soprattutto nel discorso diretto, di citazioni, aforismi (di uso quotidiano o di matrice savante), metafore, similitudini ed altri espedienti dello stile figurativo, contribuisce a tratteggiare un efficace tableau de mours sociali, dando consistenza psicologica a devisants e personaggi.

Nell'VIII ed ultimo capitolo la studiosa cerca di illustrare la teoria del linguaggio che emerge dalle opere narrative di Noël Du Fail ed in particolar modo dai discours di Eutrapel e compagni. Emblematico è in proposito il trattamento della matière gauloise. Noël Du Fail, infatti, dans le sillage di Rabelais, è uso avvalersi con garbo ed inventiva dgli accorgimenti tradizionali della comicità popolare (che disdegna di sottoporre a moralistiche censure) e della iocositas umanista: i bons mots, i calembours, l'onomastica burlesca, le ripetizioni, le enumerazioni ecc. L'effetto umoristico più riuscito scaturisce, come s'è visto, dal contrasto tra la prosaicità dei significati e il registro aulico dei significanti (e viceversa). Secondo $\mathrm{M}^{\mathrm{me}}$ Bichard-Thomine il ricorso ad uno stile prolisso ed involuto, laborioso e carico di ripetizioni e di redoublements non sarebbe, pertanto, 
una tara congenita della sua scrittura - magari connessa con la formazione eminentemente giuridica - ma un artificio volontario, con aperte finalità parodistiche, riconducibile alla polemica erasmiana contro gli eccessi e le doppiezze del linguaggio. L'arroganza di studenti boriosi o di borghesi anoblis, le smancerie di cortigiani leziosi e le elucubrazioni ingannevoli di medici ed alchimisti sono il bersaglio della sua satira feroce. Sorretta da un solido pragmatismo (che si sottrae abilmente alle pastoie speculative del Cratilismo), la sua personale filosofia del linguaggio avversa ogni forma di adulterazione linguistica, ivi compresi i prestiti ai lessici stranieri (Italiano in primis). Dietro il conservatorismo provinciale si cela l'aspirazione esistenziale dell'umanista di campagna alla naturalezza ed alla spontaneità.

Allo studio dettagliato dell'opera di Noël Du Fail fa seguito una corposa appendice di oltre $200 \mathrm{pp}$. Queste concordances dufailiane, oltre a documentare capillarmente scelte narrative, linguistiche e letterarie del gentiluomo bretone si rivelano uno straordinario strumento di ricerca per il lettore e lo studioso, al pari dell'esaustiva bibliografia che conclude questo lavoro encomiabile.

11 Dopo aver illustrato con dovizia di particolari i notevoli pregi del volume di $\mathrm{M}^{\mathrm{me}}$ Bichard-Thomine, consegnamo alle ultimissime righe della nostra recensione alcuni rilievi critici, nel dichiarato intento di offrire ulteriori spunti alla riflessione. Innanzitutto è nostro parere che la studiosa, per l'umanissima passione che ci lega ai nostri autori prediletti, abbia forse sopravvalutato la figura intellettuale del modesto giurista di provincia Noël Du Fail, rapportando, non di rado con palesi forzature, le sue scelte ideologiche e letterarie all'ascendente, non sempre riconoscibile di Rabelais e, a maggior ragione, di Erasmo. Inoltre, forse per il taglio eminentemente formale della sua ricerca, ovvero per non ripercorrere sentieri già battuti, ella ha sorvolato, a volte con eccessiva leggerezza, il contesto storico e sociale in cui l'opera e il suo autore nobiluomo terriero e parlamentare bretone - si collocano. Ridimensionando il contributo della cultura umanista nella formazione dello scrittore Noël Du Fail e considerando con la dovuta attenzione i fattori extraletterari che incidono sulle sue scelte artistiche e compositive, si può forse rileggere differentemente - e quindi sotto il segno dell'ideologia e dell'engagement, e non già della natura e della libertà - l'opera letteraria del signore della Hérissaie. Così la polifonia, in cui s'è voluto, a ragion veduta, riconoscere il modo compositivo dominante della raccolta dufailiana, rifletterebbe, non già l'eco della lezione erasmiana e rabelaisiana, ma le contraddizioni esplosive di un'epoca tormentata e conflittuale. E allora l'inane conato dell'indefesso hobereau bretone mirerebbe ad un'improbabile, ed apertamente reazionaria, reductio ad unum, rilevabile nel tentativo, non sempre felice, di conciliare narrazione e riflessione.

Ma la materia narrativa, per lo più ridotta alle dimensioni e al ruolo di mero exemplum della dottrina politica e sociale, finisce a volte per sfuggire di mano allo scrittore (che si sottrae di buon grado alle onerose mansioni del polemista), conformemente ad una tendenza - illustrata da eminenti studiosi del calibro di Kasprzyk, Sozzi e Pérouse comune a molti prosatori brevi tardo-cinquecenteschi. Ristabilito l'ordine, dopo le intemperanze del conflitto Civile, dal composto instabile del dicours bigarré si sarebbero, allora, forgiati i generi modernissimi della prosa di riflessione e del romanzo. 